\title{
Malato e medico in dialisi: un approccio alla bioetica
}

\section{Mario Timio}

\author{
Dipartimento di Medicina Interna e Dialisi, Ospedale Foligno, Foligno (PG) \\ Coordinazione Centro di Bioetica della Regione Umbria, Perugia
}

\begin{abstract}
STATUS OF ETHICS IN DiALYSIS: THE CHALLENGE OF PATIENTS AND PHYSICIANS
Abstract. According to the "Hume law", no drop of ethics can derive from a scientific rule. This law can be also applied as a guide for healthcare policy, given the limitations of secular moral reasoning. Such an approach is offered to provide the strongest case for the robust web of moral obligations in the dialysis area. It is important to inquire dialysis patients on how they wish to be treated when competent, who should make judgments on their behalf, and the circumstances under which treatment should be withdrawn. Against a plurality of visions of justice, fairness, and rights in healthcare and, in particular, in the approach to dialysis, basic moral rules of thumb can still be articulated to guide physicians, other healthcare professionals, and care-policy makers. These rules of thumb can be stated in the following issues: (i) principle of truth; (ii) principle of value and dignity of man; (iii) principle of freedom; (iv) informed consent; (v) principle of justice; (vi) bioethical models.
\end{abstract}

Key words: "Hume law", Ethics, Dialysis, Moral framework

Conflict of interest: None.

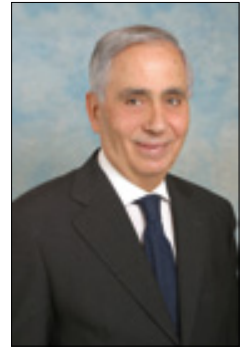

\section{La "legge di Hume" applicabile in Nefrologia}

Con la "legge di Hume" si sostiene che è logicamente impossibile derivare asserti prescrittivi da proposizioni descrittive. Il filosofo scozzese afferma che, in alcuni sistemi di etica, si incontrano delle proposizioni che esprimono un dovere senza indicare un essere e senza apportare una Mario Timio ragione adeguata. Si deduce, cioè, che un'etica non ha nulla a che fare con la scienza. In altre parole, da un asserto scientifico non si estrae una stilla di morale (1). La scienza e l'etica orbitano in ambiti diversi, anche se asserzioni indicative e asserzioni prescrittive tra fatti e valori possono non escludersi a vicenda. Ciò avviene anche nel campo della medicina e, ancora di più, nella ristretta area della Nefrologia. Con questa rubrica ne vedremo i contorni, partendo spesso dalla descrizione di situazioni reali della quotidiana attività assistenziale.

Un giorno, uscendo dalla sala dialisi, Tommaso per l'ennesima volta in breve tempo, mi sussurra: "Ho 56 anni e vivo nel mondo della dialisi dall'età di 16 anni. Dialisi peritoneale, emodialisi ospedaliera, emodialisi domiciliare, trapianto renale andato male, quindi di nuovo emodialisi e secondo trapianto ancora fallito. Rientro in dialisi. Ormai sono un rude- re. Ho tutte le complicazioni del mondo. Che altro vuole da me Dio, se esiste? E, se esiste, chiedo perché è così cattivo con me. E, se esiste e non può fare niente per me, almeno mi faccia morire. Mi creda, Dottore, non ce la faccio più a soffrire tanto".

Nelle parole di Tommaso c'è un compendio di etica del malato e del medico. In lui non c'è alcuna pedagogia dell'accettazione della sua condizione né del dolore che lo tormenta, ma solo un sussurro di ribellione e di sdegno, un invito alla sfida e alla lotta alla sopportazione, fino a chiedere la morte, se non c'è più nulla da fare, perché la sofferenza è troppo grande. Ormai avverte il suo dolore non necessario, inutile (2). La storia di Tommaso acquista nei suoi contorni rivestimenti drammaticamente umani: può fare domande, suppliche e persino lanciare anatemi come un novello Giobbe. Eppure, nella crisi dell'uomo e nel silenzio di Dio, trova le forze, forse le ultime, per l'invocazione di una risposta che dia senso non solo alla sua contestazione ma a ogni tentativo di decifrazione.

Facendo riferimento a un non-condiviso termine di coniazione sociologica, i dializzati sono spesso etichettati come "uomini marginali", sono, cioè, sospesi in un limbo posto tra il mondo dei malati e quello dei sani e, pur non appartenendo totalmente a nessuno dei due, fanno parte di entrambi (3). Però, lasciamo alla sociologia questa definizione: per noi sono uomini come gli altri, titolari di rispetto e dignità, richiedenti comprensione e aiuto. Anche perché i medici spesso inviano messaggi con- 
traddittori. Con quale bagaglio etico dovrebbero rivolgersi, per esempio, a dializzati come Tommaso? Dovrebbero dirgli: "Continua e noi saremo al tuo fianco". Ma, se non accetta di continuare, che fare? E qui balza agli occhi nella sua interezza il dilemma bioetico del dottore che, ripeto, può suggerire indicazioni contrastanti. Si consiglia di condurre una vita normale, ma, nel contempo, si raccomanda di ridurre l'apporto di liquidi a una quantità appena necessaria per ingoiare la manciata di farmaci che i pazienti devono assumere quotidianamente. Si stimola a ritornare al lavoro, ma alcuni dializzati non possono permettersi di saltare una delle tre/quattro sedute dialitiche settimanali. Sono invitati a non polarizzare l'attenzione sul loro stato di salute, ma accusano con disappunto una graduale attenuazione della forza muscolare, della potenza sessuale e della lucidità mentale, che può limitare sensibilmente la loro capacità di lavoro proficuo e di adeguata relazione sociale. I parenti, anche su consiglio del personale sanitario, spesso assumono un atteggiamento iperprotettivo e ossessivo, supervisionando qualsiasi attività o iniziativa del paziente, il che contribuisce ad accentuare il suo stato di autocommiserazione, di nichilismo e negativismo e di depressione, che talvolta sfocia nel tentato suicidio. O nel suicidio. Ogni centro dialisi ha avuto l'esperienza del suicidio di qualche paziente. Sarebbe interessante analizzare lo stato psicologico di ognuno e vedere quanto grande e totale sia stato il supporto della struttura, volto a evitare, nell'ambito del possibile e dell'imprevisto, una tragica fine. E, soprattutto, quante note di bioetica sono state messe tra parentesi, se mai ciò possa essere avvenuto.

\section{Qualità della vita oltre la sopravvivenza}

Continuando su questo argomento, il male non è solo la sofferenza (talvolta diversa dal dolore) insopportabile (quello di Tommaso, per intenderci). È anche la degradata qualità di una vita che il dializzato ritiene non degna di essere vissuta, soprattutto quando si interrompono i rapporti con gli altri e la capacità di conoscenza e di relazione, di sentimenti e di esperienza. Le scelte conseguenti al diritto e all'autonomia della persona, anche se non condivise, vanno sempre rispettate in un background relazionale e dialogico (4).

Ma non tutti i dializzati reagiscono come Tommaso. C'è, per esempio, Paolo, in dialisi da circa tre lustri, che un giorno mi fa scivolare sulla mano un biglietto. "La mia è una sorta di sfida a un destino che vorrebbe annientarmi. Dializzato va bene, ma poi amputato del piede e della gamba prima a destra e poi a sinistra. Quindi la protesi. Ora mi muovo con la sedia a rotelle. Ma continuo a sorridere alla vita. Malgrado tutto. E la fede non c'entra niente". Nella sua ottimistica accettazione, Paolo perde la dimensione eroica, acquisendo una valenza positiva e umana, che suscita empatia, vicinanza e condivisione. Al di là di ogni compassionevole pietismo. Egli non crede che la fede gli conferisca una marcia in più e non accetta che il dolore abbia valenze positive e salvifiche. Piuttosto, ha fatto proprio il principio secondo il quale il migliore atteggiamento è costruire un'immunità interiore tale da non essere intaccata dalle vicissitudini della vita. Rimanere distaccati dalla malattia e dal dolore, allenarsi a vivere con coraggio. È duro, ma è quanto insegna la moderna bioetica laica, anzi una bioetica alla rovescia emanata dalla carne sofferente verso chi questa carne cerca di curarla (5). Nelle parole di Paolo, si nasconde la gioia di vivere "malgrado tutto", di amare, di valorizzare la propria esistenza anche se monca e tribolata, poiché nulla accade senza ragioni. Egli vuole continuare a sorridere alla vita, nel suo distillato di quotidiana accettazione e di prorompente vitalità al di sopra di effervescenti emozioni e di decadenti catastrofismi. E tanti Nefrologi sanno, nel loro quotidiano contatto con i dializzati, che Paolo non è un'isola.

\section{Le attrezzature bioetiche del Nefrologo}

Diversamente, chi può spiegare il grande entusiasmo di tanti dializzati a partecipare al raduno ciclistico organizzato a Firenze in occasione del Congresso Nazionale 2013 della SIN, sullo stesso percorso dei corridori partecipanti al campionato del mondo di ciclismo nel medesimo anno? La loro forza era difficilmente attribuibile alla terapia con eritropoietina. C'era ben altro: la loro testimonianza, che ho raccolto partecipando allo stesso raduno, per dimostrare al mondo di essere uguali agli altri con le stesse motivazioni e con le stesse aspettative. Sì, anche questa è una manifestazione di bioetica alla rovescia che tante cose insegna al Nefrologo di oggi.

Ma poi c'è l'etica che attiene alla qualità della vita. Un'etica che impone di considerare l'anima insieme al sanguinamento del malato, la visione del mondo e lo stato sociale come la chiusura della fistola artero-venosa, i ritmi lavorativi come l'efficienza dialitica (6). Insomma, il malato come persona nella sua globalità. "Ma questo già lo consideriamo", possono argomentare alcuni Nefrologi. Bene! Questi colleghi hanno realizzato, forse in virtù di una sopita ma solida coscienza etica, che in poche condizioni patologiche come quella renale avvengono trasformazioni così rilevanti non solo nel funzionamento organico, ma in tutta la sfera della vita della persona. Vengono coinvolti l'essere fisico, l'essere relazionale, l'essere emozionale e l'essere spirituale. Come risponde l'operatore sanitario a ognuna di queste componenti? Come affronta le esigenze della vita affettiva? Come infonde fiducia quando il malato scivola in una voragine di disgregazione? In che modo aiuta a ritrovare un valore della propria esistenza, nonostante il cambiamento somatico e, talvolta, spirituale? L'operatore sanitario è attrezzato per queste nuove competenze al di là di quelle strettamente tecniche e scientifiche? In che modo plana sulla sua psiche, quando il dializzato non accetta la sua malattia né la terapia $o$, al contrario, si abbandona in una relazione di dipendenza morbosa con l'operatore? In ogni caso, è sempre sofferenza che si rifà, volenti o nolenti, a categorie di bioetica. Allora diventano prioritari l'ascolto della sofferenza, della condizione della paura e dell'ansia, delle difficoltà, delle fughe e dei silenzi e l'interesse per il vissuto, per le pene e le gioie, per i progetti e per i sogni del paziente. Talvolta, anche per le insofferenze e le ribellioni. Un mio paziente ce l'aveva con il mondo e imprecava sempre contro tutti per un unico motivo: perché lui doveva essere dializzato per vivere e gli altri no? E morto disperato e ogni tentativo per fargli minimamente accettare la sua condizione è miseramente naufragato. Non 
accettava nessun paternalismo né, tantomeno, nessuna autorevolezza. La sua odissea, come quella di tanti altri dializzati, spinge a chiedere la sostituzione dell'autorevolezza basata su convinzioni personali con un supplemento di etica della responsabilità, intesa come fondamento psicologico ed etico della professione e come disponibilità a mettere in discussione il proprio modo di operare, al fine di migliorare l'outcome delle prestazioni e di evitare sofferenze inutili.

\section{Conclusioni}

In sintesi, le decisioni sul trattamento dialitico comportano il riferimento a una serie di principi bioetici così riassumibili (7). a) Principio di verità. Il rapporto medico-malato deve basarsi su un dialogo fatto di ascolto, di rispetto e di considerazione della componente fisica e psicologica in cui l'aiuto a superare la difficoltà del momento è prioritario. Deve essere un incontro autentico fra uomini liberi. Ciascuno dei due partner del processo terapeutico ha una propria coscienza che non va tradita, e questa deve essere continuamente aperta a quella dell'altro e rispettata. b) Principio del valore e della dignità della vita. La vita è intesa come valore "trascendente", a prescindere dalla propria weltanschauung: la vita dell'uomo trascende la sua stessa realtà personale. Non essendo lui a darsela. Non essendo lui a potersela togliere. Ne derivano tre principi alla base di ogni valore bioetico (8): 1) deontologia kantiana. C'è una forte inflessibilità a bypassare il diritto e la santità della vita che superano ogni altra riflessione; 2) approccio esistenzialista. Si pone al centro di ogni pratica medica il consenso informato che mai come nei nefropatici ha un valore di elevato contenuto etico; 3 ) analisi utilitaristica. Si considerano i possibili trattamenti al fine di identificare quello che offre maggiori garanzie di miglioramento della qualità della vita, coniugato al massimo beneficio personale.

c) Principio della libertà. Si pone grande attenzione all'autonomia del paziente, riconoscendo che una persona in grado di prendere decisioni ha anche il diritto di scegliere di non essere curata. La responsabilità etica degli operatori sanitari è di assicurarsi che il paziente, sia giovane che adulto, abbia sufficienti informazioni e capacità discriminative, tali che sia in grado di esprimere un consenso sulle procedure mediche adottate. Sotto questo principio ritorna il costrutto del consenso informato. d) Principio di giustizia. Richiede che i pazienti siano trattati imparzialmente. Nel contesto della problematica della dialisi, questo principio si trasforma in quello di giustizia distributiva, facendo sorgere due interrogativi: quali sono i criteri di inclusione e chi deve farne le spese? Quando le risorse economiche e di disponibilità di attrezzature sono limitate, rispondere a queste domande diventa difficile. In Italia ancora di più dopo l'emanazione della spending review.

e) Modelli bioetici. Sono quelli che influenzano la pratica clinica della dialisi. Possono essere diversi e basati su un approccio che considera il trattamento come lotta alla malattia, come ruolo familiare dell'operatore che agisce per il bene del paziente, come patto tra due persone di cui una eroga un servizio e l'altra lo riceve, come alleanza di due persone, basata sul sentimento di condivisione. È di grande importanza etica per la relazione terapeutica quest'ultimo modello, nel quale dializzato e medico sono alleati e, insieme, coinvolti nelle decisioni che contano (9).

\section{Riassunto}

Secondo la "legge di Hume" non esiste una goccia di etica che può derivare da una regola scientifica. Tale legge è applicabile ad ogni ambito della scienza, compresa la scienza nefrologica. In dialisi i problemi di bioetica sono molteplici, non ultimi l'inizio della terapia sostitutiva, la scelta del tipo di dialisi, l'autonomia del paziente, il costo della pratica e la sospensione del trattamento. Spesso i problemi clinici e bioetici possono comunque embricarsi. Per favorire la convergenza delle due componenti si fa riferimento ad alcuni punti nodali: 1) principio di verità; 2) principio del valore e della dignità dell'uomo; 3) principio della libertà; 4) problema del consenso informato, 5) principio di giustizia; 5) modelli bioetici che influenzano la pratica clinica.

Parole chiave: "Legge di Hume”, Etica, Dialisi, Struttura morale

Dichiarazione di conflitto di interessi: L'Autore dichiara di non avere conflitto di interessi.

\section{Indirizzo degli Autori:}

Prof. Mario Timio

Via XX Settembre 22

06121 Perugia

timma@libero.it

\section{Bibliografia}

1. Hume D. A treatise of human nature. Selbi-Bigge. Oxford 1888; Libro I, parte 1,1.

2. Malacrida R. Il dolore non necessario. Janus, medicina, cultura, culture. Zadig Editore, Roma 2001; 1: 8-10.

3. Timio M. Il limbo degli uomini con il rene artificiale, Timio M., Classi sociali e malattie. Coines Edizioni, Milano 1976; 109-17.

4. De Santo NG, De Santo RM, Perna AF, Bellinghieri G, Cirillo M. Survival is not enough--quality of life in CKD. J. Nephrol 2008; 21 (Suppl. 13): S1-2.
5. Kmiusal Low J, et al. The impact of end-stage disease and close person. A literature review. Nephrol Dial Transplant 2008; 2: 67-9.

6. Eady RA. Survival is not enough: reflections of a long-term renal patient. J Nephrol 2008; 21 (Suppl. 13): S3-6.

7. Russo G. Bioetica Medica. Per medici e professionisti della sanità. Coop. S. Tommaso 2009, Messina; 325-30.

8. Kjellstrand CM, Dossetor JB. Ethical problems in dialysis and transplantation. Kluwer Academic Publishers Dordrechet/Boston/London 1992; 106-10.

9. Timio M. Codice di deontologia medica, in Di Pilla F. (a cura di). Seminario di Bioetica. Aracne, Roma 2008; 293-304. 